\title{
Demonstration and evaluation of a method for assessing mediated moderation
}

\author{
ANTONIO A. MORGAN-LOPEZ \\ Research Triangle Institute, International, Research Triangle Park, North Carolina \\ and \\ DAVID P. MACKINNON \\ Arizona State University, Tempe, Arizona
}

\begin{abstract}
Mediated moderation occurs when the interaction between two variables affects a mediator, which then affects a dependent variable. In this article, we describe the mediated moderation model and evaluate it with a statistical simulation using an adaptation of product-of-coefficients methods to assess mediation. We also demonstrate the use of this method with a substantive example from the adolescent tobacco literature. In the simulation, relative bias (RB) in point estimates and standard errors did not exceed problematic levels of $\pm 10 \%$, although systematic variability in RB was accounted for by parameter size, sample size, and nonzero direct effects. Power to detect mediated moderation effects appears to be severely compromised under one particular combination of conditions: when the component variables that make up the interaction terms are correlated and partial mediated moderation exists. Implications for the estimation of mediated moderation effects in experimental and nonexperimental research are discussed.
\end{abstract}

Mediational analyses are used to assess the degree to which a variable is intermediate in the causal sequence between a predictor and a dependent variable. The relation between two variables may also depend on another variable, called a moderator variable (Baron \& Kenny, 1986). Moderator variables affect the strength of a relation between two variables and are typically tested with interaction effects. In some situations, moderators and mediators operate simultaneously. Mediated moderation, for example, occurs when the interaction between two variables affects a mediator, which then affects a dependent variable (Baron \& Kenny, 1986; Donaldson, 2001). The purposes of this article are to combine methods developed separately for mediation analysis and moderation analysis in a mediated moderation model, evaluate the model with a statistical simulation, and illustrate a practical example of this method with real data.

\section{Mediation}

Mediational analyses identify cognitive or behavioral processes that relate an independent variable to a depen-

This research was supported by NIMH Grant 2T32MH18387, NIDA Grant 5R01DA09757-04, and the Center for Interdisciplinary Substance Abuse Research, RTI International. We acknowledge the contributions of Matthew Fritz, Chondra Lockwood, Aaron Taylor, and Stephen G. West to this work. Portions of this research were presented at the 9th annual meeting of the Society for Prevention Research, June 2001, Washington, DC. Correspondence concerning this article should be addressed to A. A. Morgan-Lopez, Center for Interdisciplinary Substance Abuse Research, Research Triangle Institute, International, 3040 Cornwallis Road, Research Triangle Park, NC 27709 (e-mail: amorganlopez@rti.org). dent variable. Mediational processes guide the development and evaluation of preventive intervention trials (Lipsey, 1993; MacKinnon \& Dwyer, 1993; MacKinnon, Taborga, \& Morgan-Lopez, 2002; West \& Aiken, 1997). In etiological studies, mediation analyses help identify links between risk factors and outcomes (see, e.g., MacKinnon et al., 1991; Wolchik et al., 1993).

In program evaluation, mediational analyses provide practical information about the success or failure of action theory and the conceptual theory used in the development of the program (Chen, 1990). Action theory refers to the relation between program components and the mediator(s) that the program is designed to change. The conceptual theory refers to the relation between the mediator(s) and the outcome variable. Through mediational analysis, researchers can evaluate whether or not a program was successful in changing the mediating variable that it was designed to change (action theory) and whether or not the mediating variable changed the outcome variable (conceptual theory). More importantly, mediation analyses can identify which program components and mediators were or were not responsible for transmitting the program effects (Donaldson, 2001; MacKinnon, Taborga, \& MorganLopez, 2002).

The advantages of the mediational model are numerous and well documented in comparison with the analysis of the simple $X \rightarrow Y$ relationship (Baron \& Kenny, 1986; Donaldson, 2001; Lipsey, 1993; MacKinnon, 1994; MacKinnon, Taborga, \& Morgan-Lopez, 2002). However, there are important questions that mediational analyses cannot answer. Whereas mediational analyses can provide information about mediational processes, they cannot pro- 
vide information about whether or not these processes differ across subpopulations.

\section{Moderation}

Moderator analyses evaluate whether or not a relation between two variables may differ in direction or magnitude across subpopulations or settings (Baron \& Kenny, 1986). That is, moderator analyses test whether or not the relation between $X$ and $Y$ changes as a function of a third variable, $Z$ (Aiken \& West, 1991; Baron \& Kenny, 1986; Donaldson, 2001). Sharma, Durand, and Gur-Arie (1981) have described more specific conceptualizations of moderation, including whether or not $Z$ serves as a predictor of $Y$ that is independent of $X$, as a pure moderator of the $X \rightarrow Y$ relationship that has no independent effect on $Y$, or in both functions.

Although moderational analyses are useful in exploring conditional relationships between predictor(s) and outcome(s), they do not address mediational processes in the relation between a predictor and a dependent variable (Donaldson, 2001; MacKinnon, 1994). Thus, mediational models in which moderators are also examined may provide more information than either statistical approach alone. These include cases in which the moderator variable, $Z$, moderates the effect of the predictor on the mediator (e.g., $X \rightarrow M$ ) or that of the mediator on the outcome (e.g., $M \rightarrow Y$; Baron \& Kenny, 1986; Donaldson, 2001).

\section{Mediation and Moderation}

The combination of mediation and moderation approaches may be especially useful in studying the etiology and prevention of negative health outcomes (Tein, Sandler, \& Zautra, 2000; Wolchik et al., 1993). Etiological research often focuses on factors that mediate the relationship between a risk condition and a health outcome. However, the mediational process by which risk influences outcome may be moderated by other risk factors or buffered by protective factors (Donaldson, 2001; Morgan-Lopez, Castro, Chassin, \& MacKinnon, 2003).

In program evaluation, combining mediation and moderation analyses may identify variables that either compromise or improve the efficacy of an intervention (MacKinnon, Taborga, \& Morgan-Lopez, 2002; Wolchik et al., 1993). Similarly, mediation and moderation analyses help identify subpopulations that do not benefit from an intervention and inform cost-benefit decisions such as which groups to target with an intervention (Donaldson, 2001; MacKinnon, Taborga, \& Morgan-Lopez, 2002; West \& Aiken, 1997). In short, mediated moderation (and moderated mediation) analysis assesses the generalizability of the mediated effect.

Mediation and moderation have been combined in several areas of research. Wolchik et al. (1993) evaluated mediated effects of an intervention for divorced mothers on child behavior problems. Differences among the mediated effects of the program were tested as a function of baseline child behavior problems. The mediated effects did not vary across children who had low versus high levels of child behavior problems at baseline (Wolchik et al., 1993).
Tein et al. (2000) examined whether or not psychological distress mediated the effects of stressful events on parenting quality and the extent to which coping strategies moderated the influence of distress on parenting quality in a sample of divorced mothers. The model described in Tein et al. (2000) is a moderated mediation model (Baron \& Kenny, 1986; Donaldson, 2001; James \& Brett, 1984).

Mediated moderation and moderated mediation models are distinguished on the basis of which individual path in the mediational chain varies as a function of the moderator variable. In the mediated moderation model, the path from the intervention to the mediator (i.e., $X \rightarrow M$ ) depends on the level of a moderator variable, $Z$, whereas the effect of the mediator on the outcome (i.e., $M \rightarrow Y$ ) is constant. In moderated mediation models, the path from the intervention to the mediator (i.e., $X \rightarrow M$ ) is constant, whereas the effect of the mediator on the outcome (i.e., $M \rightarrow Y$ ) depends on the level of $Z$.

Mediated moderation models, the subject of the present study, involve the interaction between two predictor variables on a mediator, which, in turn, affects an outcome. Unger et al. (2000) investigated mediators of the effect of acculturation status on cigarette use among Latino and Asian-American youth. They also tested for moderation in these mediational processes as a function of subgroup differences among the Asian-American sample (e.g., country of origin). Morgan-Lopez et al. (2003) found that the protective influence of Mexican cultural norms on cigarette use was mediated by youths' confidence in their ability to avoid tobacco. However, this mediated effect was diminished, albeit still significant, in the presence of peer smoking influences on youth confidence.

\section{Estimation of Mediated Moderation}

Equations 1 and 2 depict the mediation model described elsewhere (MacKinnon \& Dwyer, 1993):

$$
\begin{aligned}
M & =\iota_{1}+\alpha X+\varepsilon_{1}, \\
Y & =\iota_{2}+\beta M+\tau^{\prime} X+\varepsilon_{2},
\end{aligned}
$$

where $X$ is the independent variable, $M$ is the mediator, $Y$ is the outcome variable, $\alpha$ codes the regression of $M$ on $X$, $\beta$ codes the regression of $Y$ on $M$ adjusted for $X, \tau^{\prime}$ codes the regression of $Y$ on $X$ adjusted for $M, \iota_{1}$ codes the predicted value of $M$ when $X=0, \iota_{2}$ codes the predicted value of $Y$ when $X=M=0, \varepsilon_{1}$ codes the difference between observed and model-predicted scores on $M$, and $\varepsilon_{2}$ codes the difference between observed and model-predicted scores on $Y$. The model assumes that the expected value of the residual terms $\left(\varepsilon_{1}, \varepsilon_{2}\right)$ equals 0 , the covariance among the residual terms $\left(\varepsilon_{1}, \varepsilon_{2}\right)$ equals 0 , and model-based residuals are independent of all predictors. The product, $\alpha \beta$, is the mediated effect.

In the mediated moderation model (see Figure 1), Equations 3 and 4 expand on the mediation model to include an additional predictor, $Z$ :

$$
\begin{aligned}
M & =\iota_{1}+\alpha_{1} X+\alpha_{2} Z+\alpha_{3} X Z+\varepsilon_{1}, \\
Y & =\iota_{2}+\beta M+\tau_{1}^{\prime} X+\tau_{2}^{\prime} Z+\tau_{3}^{\prime} X Z+\varepsilon_{2},
\end{aligned}
$$


where $X$ is the independent variable; $Z$ is an independent variable/moderator; $X Z$ is the interaction of $X$ and $Z ; M$ is the mediator; $Y$ is the outcome variable; $\alpha_{1}$ codes the regression of $M$ on $X$ adjusted for $Z$ and $X Z ; \alpha_{2}$ codes the regression of $M$ on $Z$ adjusted for $X$ and $X Z ; \alpha_{3}$ codes the regression of $M$ on the $X Z$ interaction adjusted for $X$ and $Z$, and tests the null hypothesis that the regression of $M$ on $X$ is consistent across levels of $Z$ (Aiken \& West, 1991); $\beta$ codes the regression of $Y$ on $M$ adjusted for $X, Z$, and $X Z ; \tau_{1}^{\prime}$ codes the regression of $Y$ on $X$ adjusted for $M$, $Z$, and $X Z ; \tau_{2}^{\prime}$ codes the regression of $Y$ on $Z$ adjusted for $X, M$, and $X Z ; \tau_{3}^{\prime}$ codes the regression of $Y$ on $X Z$ adjusted for $X, M$, and $Z ; \iota_{1}$ codes the predicted value of $M$ when $X=Z=X Z=0 ; \iota_{2}$ codes the predicted value of $Y$ when $X=M=Z=X Z=0 ; \varepsilon_{1}$ codes the difference between observed and model-predicted scores on $M$; and $\varepsilon_{2}$ codes the difference between observed and modelpredicted scores on $Y$. This model also assumes that the expected value of the residual terms $\left(\varepsilon_{1}, \varepsilon_{2}\right)$ equals 0 , the covariance among the residual terms $\left(\varepsilon_{1}, \varepsilon_{2}\right)$ equals 0 , and model-based residuals are independent of all predictors.

The estimate of the mediated moderation effect is the product of the path from the interaction term $X Z$ to the mediator $M, a_{3}$, and the path from the mediator to the outcome variable $Y, b$. This product, $a_{3} b$, reflects the extent to which the mediated effect $(X \rightarrow M \rightarrow Y)$ is conditioned on a fourth variable, $Z$. In practice, a significant mediated moderation effect would indicate the need to examine the significance of the mediated effect at various levels of the fourth variable (Aiken \& West, 1991; see also Morgan-Lopez et al., 2003; Tein, Sandler, MacKinnon, \& Wolchik, 2004).

The first illustration of mediated moderation was described by Baron and Kenny (1986) and is based on a different point estimator of the mediated moderation effect. The approach uses Equation 4 above and Equation 5 below.

$$
Y=\iota_{1}+\tau_{1} X+\tau_{2} Z+\tau_{3} X Z+\varepsilon_{3} .
$$

The mediated moderation effect is obtained by taking the difference between $\tau_{3}$ and $\tau_{3}^{\prime}$ from the two equations. The $\tau_{3}-\tau_{3}^{\prime}$ estimator $\left(c_{3}-c_{3}^{\prime}\right)$ and the $\alpha_{3} \beta$ estimator $\left(a_{3} b\right)$ of the mediated moderation effect are equivalent in ordinary least squares regression (see MacKinnon, Warsi, \& Dwyer, 1995).

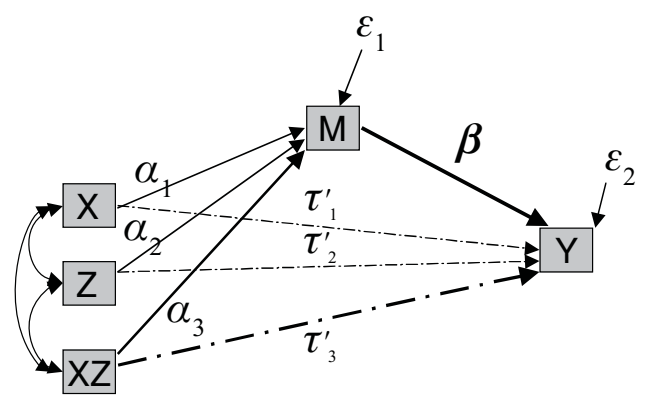

Figure 1. Mediated moderation model.

\section{Sampling Distribution of the Product}

The mediated effect is equal to the product of the parameter estimates $a$ and $b$ for $\alpha$ and $\beta$ from Equations 1 and 2. The variance of the product of $a$ and $b$ was derived by Sobel (1982) using the multivariate delta method (Bishop, Fienberg, \& Holland, 1975), a general method for deriving the variance of functions of random variables. The multivariate delta method estimator for the variance of $a b$ is $\left(a^{2} s_{b}^{2}+b^{2} s_{a}^{2}\right)^{1 / 2}$ (Baron \& Kenny, 1986; MacKinnon et al., 1995; Sobel, 1982), where $a$ is the estimate linking the predictor to the mediator, $b$ is the estimate linking the mediator to the outcome, and $s_{a}^{2}$ and $s_{b}^{2}$ are the $S E$ s for the respective effects. For the mediated moderation model, the estimate $a$ is substituted by the estimate $a_{3}$, the sample value for the $\alpha_{3}$ parameter in Equation 4.

One of the more appealing features of the $a b$ method is that the estimates used for the analysis, $a$ and $b$, represent direct estimates of the success or failure of both the action theory and the conceptual theory links, respectively, in the evaluation of a program (MacKinnon, Taborga, \& MorganLopez, 2002) or an etiological process. The present study will focus on this multivariate delta method $S E$ estimator (Sobel, 1982), available in several covariance structure analysis software packages (e.g., LISREL [Jöreskog \& Sörbom, 1996]; EQS [Bentler, 1995]).

Simulation studies on the multivariate delta $S E$ estimator of $a b$ have found that it has reasonable statistical properties, including negligible bias in point estimation at sample sizes of 50 or greater and negligible bias in $S E$ estimation at sample sizes above 100 (MacKinnon, Lockwood, Hoffman, West, \& Sheets, 2002). However, the performance of this and other methods of testing for mediated effects may not be generalizable to mediated moderation because of several unique aspects of the model. Specifically, covariances among the predictors, additional direct effects (e.g., $\tau_{2}^{\prime}, \tau_{3}^{\prime}$ ), an additional indirect effect (e.g., $Z \rightarrow$ $M \rightarrow Y$ ), and variances of $X$ and $X Z$ all have an influence on point estimation and confidence interval estimation for mediated moderation effects that are not present in the mediation model.

No previous studies have been conducted on the statistical properties of the mediated moderation effect. The purpose of this study is to examine the effect of sample size, effect size, and nonzero versus zero direct effects (i.e., partial vs. complete mediated moderation) on Type I error rates, statistical power to detect the mediated moderation, and relative bias (RB) in the mediated moderation effect and its $S E$.

\section{Assumptions of Mediated Moderation}

In the simulation context, model and parameter specifications are defined by the investigator. However, in the context of real data, statistical and conceptual assumptions must be either made or tested relative to the specification of any model that may not be unique to the mediated moderation model. Key statistical and conceptual assumptions are critical to the estimation of the mediated moderation 
model, particularly in nonexperimental and cross-sectional contexts (Simon, 1954). First, this model assumes that if $\mathrm{C}(X, Z) \neq 0$, then the nonzero covariance is neither the result of a causal relationship between $X$ and $Z$ nor the result of a spurious relationship between $X$ and $Z$ that can be accounted for by another variable. If $\mathrm{C}(X, Z) \neq 0$, then theoretical considerations in the substantive area of interest may make the preceding assumption tenable. Next, it is assumed that the residual terms in this model are uncorrelated with each other, suggesting that the unmeasured influences on $M$ are unrelated to unmeasured influences on $Y$. Finally, it is assumed that the residual variance(s) are constant throughout the range of each predictor.

This model also makes conceptual assumptions about causal ordering. It assumes that the causal ordering of the variables is correct such that $M$ does not cause $X, M$ does not cause $Z, Y$ does not cause $M, Y$ does not cause $X$, and $Y$ does not cause $Z$. In the absence of experimental control, logical reasoning or previous research in a particular area may provide insight into the viability of the temporal precedence assumptions made in this model. The simulation was constructed in such a way that each of these statistical and conceptual assumptions was true for the population.

\section{METHOD}

\section{Study of Type I Error}

The purpose of the first simulation study was to examine the variability in Type I error rates for this method of assessing mediated moderation effects across several conditions. SAS Version 8e (SAS Institute, 2000) was used to conduct the simulations. For each simulation, $X, Z$, and the residual variances for $M$ and $Y$ (see Equations 3 and 4 ) were generated from a standard normal distribution using the SAS RANNOR function.

Simulation conditions. The factors that varied in the study of Type I error were direct effect condition (zero or nonzero), parameter value for $\alpha_{1}(0, .14, .36$, or .51$)$, parameter value for $\alpha_{2}(0, .14$, .36 , or .51$)$, sample size $(50,100,200,500,1,000$, or 5,000$)$, the correlation between $X$ and $Z$ ( 0 or .4), parameter values for $\alpha_{3}(0$, .14 , or .36), and parameter values for $\beta(0, .14, .36$, or .51). For the simulation on Type I error, only the six combinations of $\alpha_{3}$ and $\beta$ that produced an $\alpha_{3} \beta$ product of 0 were generated and analyzed. This resulted in 2,304 combinations of parameter values and sample sizes. Five hundred replications were generated for each combination, producing a total of $1,152,000$ simulated data sets. Information from each data set, such as population parameter values, sample sizes, and inferences from the analysis of each simulated data set, was saved for analysis. The key outcome was false rejection of the null hypothesis that $\alpha_{3} \beta=0$ (Type I error). The Type I error rate (for each combination) was the proportion of false rejections of the null hypothesis out of the 500 simulated data sets.

Effect sizes for $\boldsymbol{\alpha}_{\mathbf{3}}$ and $\boldsymbol{\beta}$. In the present study, we used population parameter values for regression coefficients in Equations 3 and 4 that were derived using covariance algebra (see Appendixes A and B). Effect sizes (squared partial correlations) were calculated for each combination of population parameters used in the simulation using a matrix procedure for the calculation of (squared) partial correlations outlined in Johnson and Wichern (2002, p. 407). This allows for the comparison of simulation parameters and practical effect sizes as interpreted in applied research. For each value of $\alpha_{3}$, a range of effect sizes is described, because the effect sizes of $\alpha_{3}$ changed as the sizes of other parameters (i.e., $\beta$ ) changed. For $\alpha_{3}$, the parameter value .14 corresponded to $\rho^{2}$ values ranging from .006 to .018 , whereas a parameter value of .36 corresponded to $\rho^{2}$ values ranging from .074 to .112 . The $.14, .36$, and .51 parameter values for $\beta$ corresponded to $\rho^{2}$ values of $.019, .114$, and .206, respectively.

\section{Study of Empirical Power}

Simulation conditions. The purpose of the second simulation study was to study the factors that account for variability in empirical power. The factors that varied in the study of Type I error were direct effect condition (zero or nonzero), parameter value for $\alpha_{1}(0$, $.14, .36$, or .51$)$, parameter value for $\alpha_{2}(0, .14, .36$, or .51$)$, sample size $(50,100,200,500,1,000$, or 5,000$)$, the correlation between $X$ and $Z\left(0\right.$ or .4), parameter values for $\alpha_{3}(0, .14$, or .36), and parameter values for $\beta(0, .14, .36$, or .51). For the simulation on empirical power, only the six combinations of $\alpha_{3}$ and $\beta$ that produced an $\alpha_{3} \beta$ product greater than 0 were generated and analyzed. This resulted in 2,304 combinations of parameter values and sample sizes. Five hundred replications were generated for each combination, producing a total of 1,152,000 simulated data sets. Information from each data set, such as population parameter values, sample sizes, and inferences from analysis of each simulated data set, was saved for analysis. The key outcome in the second study was correct rejection of the null hypothesis that $\alpha_{3} \beta=0$ (power). Power for each combination was the proportion of correct rejections of the null hypothesis out of the 500 simulated data sets.

The influence of parameter values, sample size, direct effect condition, and predictor intercorrelation on $\mathrm{RB}$ in $\alpha_{3} \beta$ point estimate and $\mathrm{RB}$ in $\alpha_{3} \beta S E$ was also examined (using multiple regression). $\mathrm{RB}$ was defined as (estimated - true value)/true value. These values ( $\mathrm{RB} \alpha_{3} \beta$ and $\mathrm{RB} \sigma_{\alpha_{3} \beta}$ ) were calculated in each simulated data set. The key in these analyses was to identify combinations of conditions that would produce RB values above $\pm 10 \%$. For all analyses, sample size was converted to $\log _{10}$ in order to alleviate concerns about using a continuous predictor with a wide range of values (i.e., 50-5,000).

\section{RESULTS}

\section{Accuracy of the Mediated Moderation Effect $\left(\alpha_{3} \beta\right)$ Point Estimator}

Two factors influenced variability in the RB of the mediated moderation point estimator. The size of $\beta$ (with $S E$ expressed in parentheses) was related to RB in point estimation [ -.088 (.032), $t=2.68, p=.007]$; RB decreased as the size of $\beta$ increased. Also, direct effect condition was related to RB in point estimation [.037 (.010), $t=3.49$, $p=.0005]$; $\mathrm{RB}$ was greater when direct effects were nonzero. Each of these factors interacted with sample size, and the direct effect $\times N$ effect $[-.008(.003), t=-2.73$, $p=.006]$ and the $\beta \times N$ effect [.018 (.009), $t=2.01, p=$ $.044]$ interactions were significant. Examination of the predicted means suggested that as sample size increased, the difference in RB across $\beta$ and direct effect condition was reduced. Overall, no combination of parameter value and sample size produced an average RB value that exceeded $\pm 10 \%$, suggesting that point estimation is accurate for all conditions examined in this study.

\section{Accuracy of the Standard Error of the Mediated Moderation Effect $\left(\sigma_{a_{3} \beta}\right)$}

Several factors accounted for variability in the RB of the $S E$ of the mediated moderation effect, including the size of $\alpha_{3}[-.059(.013), t=-4.26, p<.0001]$, the size of $\beta$ [-.142 (.010), $t=-14.19, p<.0001]$, sample size [-.014 (.001), $t=-8.28, p<.0001]$, and the direct effect condition [.019 (.003), $t=5.21, p<.0001]$. RB in 
$\sigma_{\alpha_{3} \beta}$ decreased as $\alpha_{3}, \beta$, and sample size increased, respectively. RB was also higher when direct effects were nonzero. Two key interaction effects were also observed. The impact of $\alpha_{3}$ on RB depended on the direct effect condition $[-.024(.006), t=-3.48, p=.0005]$. Examination of the predicted means of RB for this effect suggests that as $\alpha_{3}$ increased in size, the difference in RB across direct effect conditions became smaller. The impact of $\beta$ on RB also depended on the direct effect condition $[-.026(.005), t=$ $-5.32, p<.0001]$. Examination of predicted means of RB for this effect suggests that as $\beta$ increased in size, the difference in RB across direct effect conditions became smaller. No combination of parameters, sample sizes, direct effect condition, and correlation between $X$ and $Z$ produced RB in the mediated moderation $S E$ that exceeded $\pm 10 \%$.

\section{Variation in Type I Errors}

Several factors accounted for the variability in Type I errors, including the size of $\alpha_{3}$ [16.10 (1.18), Wald $\chi^{2}=$ $184.19, p<.0001]$, the size of $\beta$ [20.64 (2.72), Wald $\left.\chi^{2}=57.47, p<.0001\right]$, the correlation between $X$ and $Z\left[\mathrm{C}(X, Z), 2.71\right.$ (1.11), Wald $\left.\chi^{2}=5.94, p=.014\right]$, and sample size [2.43 (.138), Wald $\left.\chi^{2}=311.87, p<.0001\right]$. Type I error rates increase as $\alpha_{3}, \beta$, sample size, or $\mathrm{C}(X, Z)$ increased. Of particular note was a significant four-way interaction between the size of $\alpha_{3}$, sample size, direct effect condition, and $\mathrm{C}(X, Z)$ on Type I error rates. In lieu of interaction contrasts and simple effect tests, qualitative trends based on the visual inspection of the observed Type I error rates are discussed on the basis of Figures 2 and 3. Visual inspection of the four-way interaction effect suggests that (1) the difference between Type I error rate trends for $\alpha_{3}=.14$ and $\alpha_{3}=.36$ (across sample size) is larger when the correlation between $X$ and $Z$ is nonzero, (2) differences in Type I error rates become smaller as sample size increases across all other conditions, and (3) the direct effect condition has the largest impact on Type I error when $\alpha_{3}=.14$ and $\mathrm{C}(X, Z)=0$. In the third case, Type I error rates may be impacted most in the context of partial mediated moderation, particularly when small interaction effects are expected in experimental research. Overall, this appears to be a conservative method of estimating mediated moderation effects, as is evidenced by Type I error rates that are consistently below the nominal rate of .05 . Type I error rates came closest to .05 as sample size approached 500 and, in many cases (i.e., with smaller values of $\alpha_{3}$ ), required samples of 1,000 or more to approach a Type I error rate of .05. This is generally consistent with findings on the use of the Sobel (1982) sampling distribution estimator in the three-variable mediation model (MacKinnon, Lockwood, et al., 2002). However, the interactive impact of direct effects and correlations between additional predictors in this study (which, to our knowledge, have not been examined elsewhere) make a comprehensive comparison with findings from other simulation studies on mediation difficult.

\section{Variation in Empirical Power}

Several factors influenced power to detect mediated moderation effects, including the size of $\alpha_{3}$ [38.52 (1.05), Wald $\left.\chi^{2}=1,324.13, p<.0001\right]$, the size of $\beta[33.65$ (.795), Wald $\left.\chi^{2}=1,791.76, p<.0001\right]$, and sample size [9.35 (.124), Wald $\left.\chi^{2}=5,671.52, p<.0001\right]$. There were two key interaction effects. The three-way interaction between direct effect condition, $\mathrm{C}(X, Z)$ and sample size was significant [1.71 (.319), Wald $\chi^{2}=28.98, p<$ $.0001]$. When simulation data are collapsed across all parameter combinations, power is similar across the direct effect condition and the $\mathrm{C}(X, Z)$ condition, except in one instance: when the correlation between $X$ and $Z$ is nonzero under partial mediated moderation (i.e., nonzero direct main and interaction effects; see Figure 4).

The four-way interaction effect between $\alpha_{3}, \beta$, sample size, and $\mathrm{C}(X, Z)$ was significant [22.51 (6.69), Wald

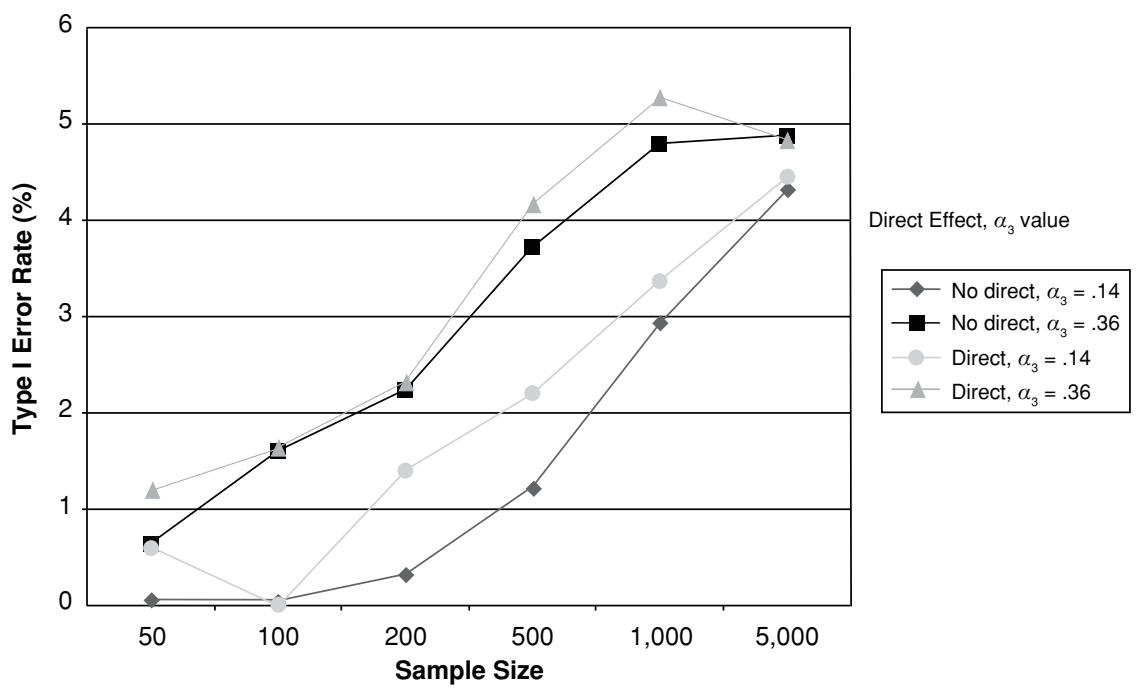

Figure 2. Empirical Type I error rate: $\alpha_{3} \times$ direct effect $\times$ sample size interaction $[C(X, Z)=0]$. 


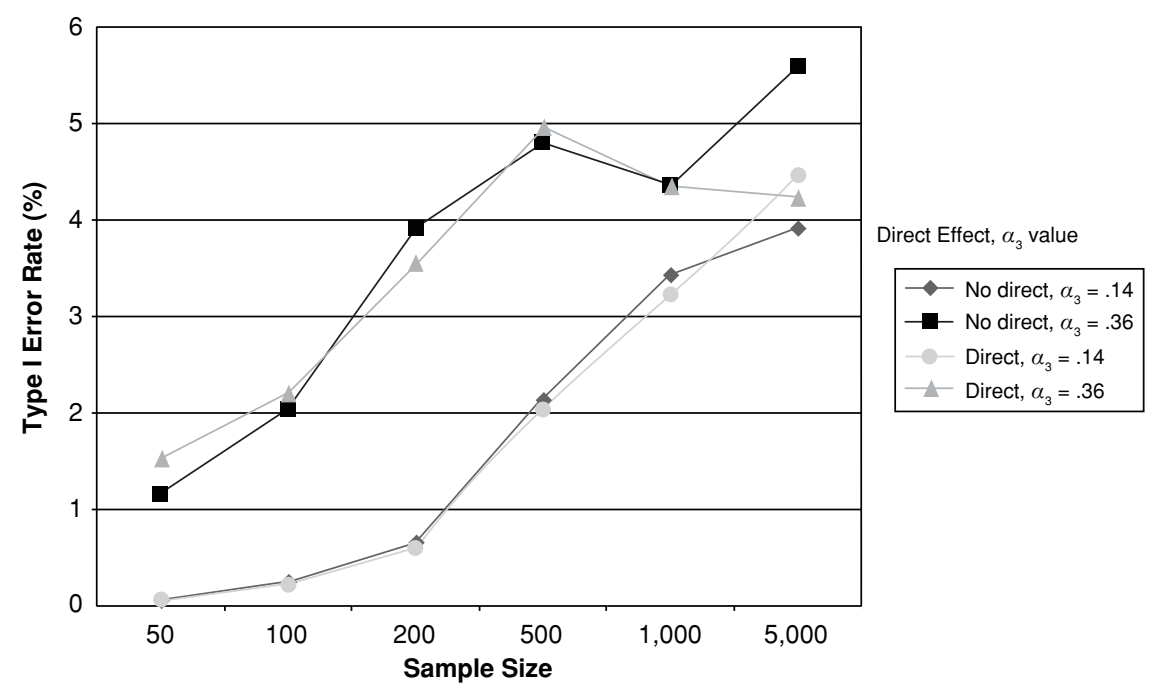

Figure 3. Empirical Type I error rate: $\alpha_{3} \times$ direct effect $\times$ sample size interaction $[\mathrm{C}(X, Z)=.4]$.

$\left.\chi^{2}=11.32, p=.0008\right]$. A comparison of Figures 5 and 6 reveals that the greatest difference in empirical power across the $\mathrm{C}(X, Z)$ conditions occurs at $N=200$ for two parameter combinations $\left(\alpha_{3}=.14, \beta=.36\right.$ and $\alpha_{3}=.14$, $\beta=.51)$. These two parameter combinations (at $N=200)$ are combinations that produce more than a $10 \%$ difference in empirical power across the two $\mathrm{C}(X, Z)$ conditions. The correlation between $X$ and $Z$ may have an impact on power only at moderate sample sizes for moderately sized mediated moderation effects.

\section{An Example of Mediated Moderation Analysis}

To illustrate mediated moderation model estimation, we use an example from Morgan-Lopez et al. (2003). This example offers an examination of the extent to which the level of investment in one's indigenous culture (i.e., enculturation status) decreases risk for cigarette use among
Mexican-American youth. The study focused on mediated effects of enculturation on cigarette use as well as contextual risk factors that were hypothesized to moderate these mediated effects. The goal of Morgan-Lopez et al.'s study was to conduct a simultaneous test of two complementary theories that account for the influence of enculturation on substance use (Castro, Coe, Gutierres, \& Saenz, 1996; Oetting, Donnermeyer, Trimble, \& Beauvais, 1998).

The theory of communal health consequences (Castro et al., 1996) suggests that youth who are invested in more collectivistic cultures should be more concerned about how their health decisions affect others, which theoretically should reduce tobacco use. This theory may be considered a "main effect" theory of protection from tobacco use, since it does not speak to differential effects of collectivism across smoking contexts. Oetting et al. (1998) suggested that levels of investment in multiple contexts

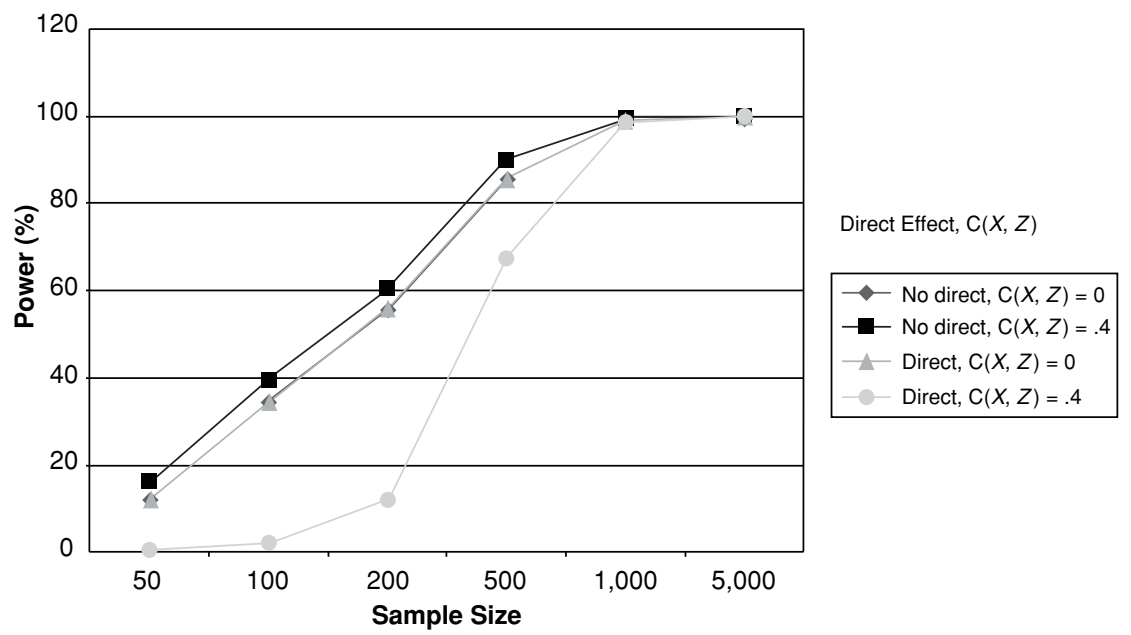

Figure 4. Direct effect condition $\times C(X, Z) \times$ sample size interaction effect on power. 


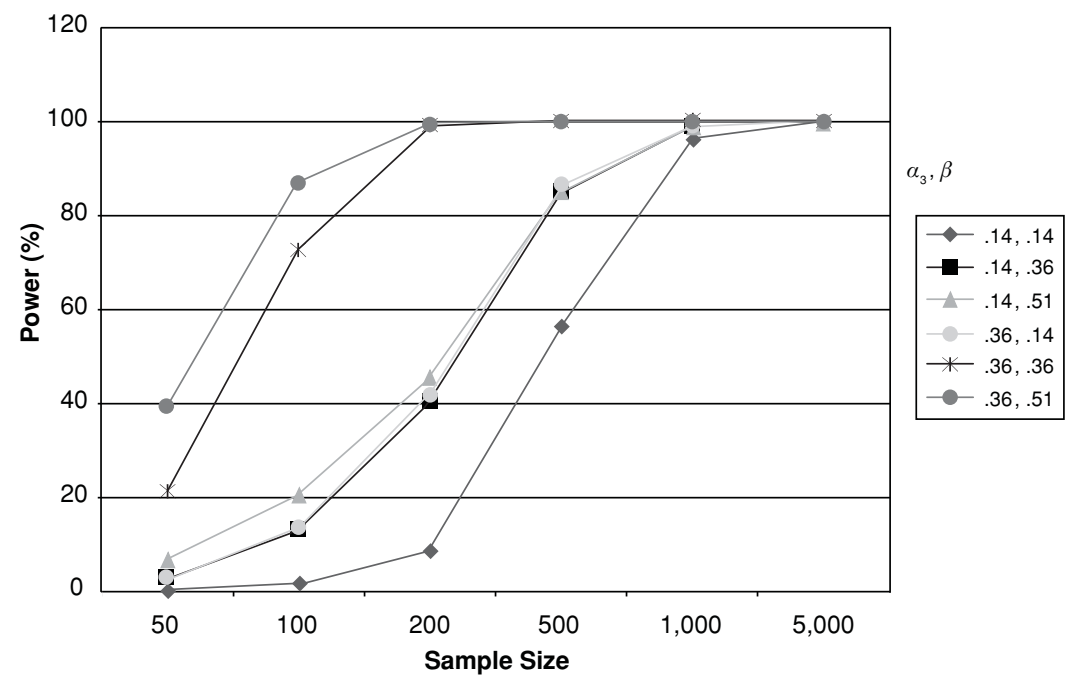

Figure 5. $a_{3} \times b \times N$ interaction effect on power; $C(X, Z)=0$.

(i.e., peer group, family, community, culture) may interact to impact the health behaviors of youth. This might suggest that youth in collectivistic cultures (which would be protective) may see lower rates of protection in high smoking contexts (which would convey risk), suggesting a moderating effect of smoking context on the relation between cultural orientation and tobacco use.

Data were from preintervention reports of 921 MexicanAmerican students in a culturally oriented tobacco prevention program (Castro, De Anda, Abeita, \& Morgan-Lopez, 1999). Forty-four percent had at least one friend who smoked, and $50.7 \%$ had at least one household member who smoked. Thirty-six percent had tried at least one cigarette in their lifetime, and $7.7 \%$ smoked at least one cigarette per day. The measures for this model are the following: number of friends who smoke (PS, single item), ethnic cultural norms (ECN; 4 items, Cronbach's alpha $=.70$ ), tobacco avoidance self-efficacy (TASE; 9 items, Cronbach's alpha $=.86$ ), and youth cigarette use (YCU; four items, Cronbach's alpha $=.89$ ). (The original analyses for these data included separate models for main mediation effects and mediated moderation effects. The present reanalysis of these data estimates main mediated effects and mediated moderation effects simultaneously. As a result, some estimates may differ from those of the original article.)

The estimate for the mediated moderation model for Equation 3 is TASE $\mathrm{predicted}=\mathrm{ECN}[2.392(S E=0.024)+$ $.239(S E=0.029)]-\mathrm{PS}[.100(S E=0.012)]-(\mathrm{ECN} \times$ PS) $[.034(S E=0.016)]$. The estimate for the mediated moderation model of Equation 4 is $\mathrm{YCU}_{\text {predicted }}=$ TASE $[.264(S E=0.074)-.276(S E=0.041)]-\mathrm{ECN}[.007$ $(S E=0.041)]+\mathrm{PS}[.267(S E=0.014)]+(\mathrm{ECN} \times \mathrm{PS})$ $[.026(S E=0.018)]$.

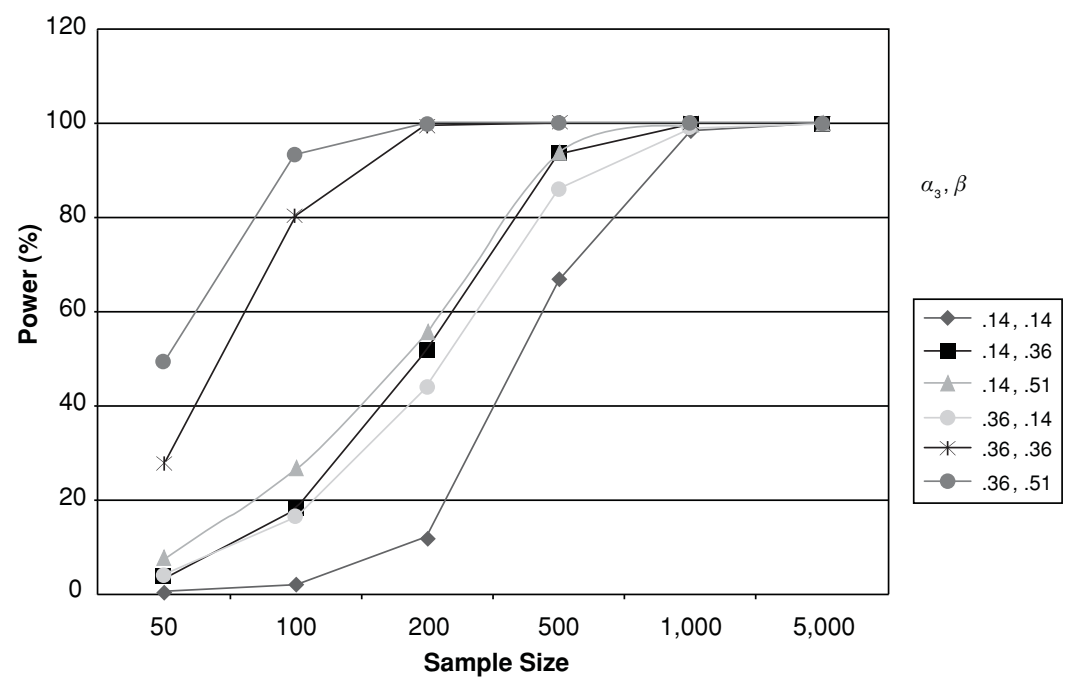

Figure 6. $a_{3} \times b \times N$ interaction effect on power; $\mathrm{C}(X, Z)=.4$. 
In testing the main mediation effect $(\mathrm{ECN} \rightarrow$ selfefficacy $\rightarrow$ YCU), the point estimate $(-.065)$ is the product of the estimates for the regression of TASE on ECN (.239) and the regression of YCU on TASE (-.276). The $S E$ of the main mediation effect in this example is .012, or $\left[\left(.239^{2}\right)\left(.041^{2}\right)+\left(-.276^{2}\right)\left(.029^{2}\right)\right]^{1 / 2}$. The results suggested that self-efficacy fully mediated the protective influence of ECN on YCU at the average level of peer smoking $\left[a_{1} b=-.065\right.$ (.012), $\left.Z=-5.21, p<.01\right]$, indicating that youth with high investment in Mexican culture had higher levels of TASE - that is, those with higher selfefficacy were less likely to smoke.

In testing the mediated moderation effect $(\mathrm{ECN} \times$ $\mathrm{PS} \rightarrow \mathrm{TASE} \rightarrow \mathrm{YCU})$, the point estimate (.009) is the product of the estimates for the regression of TASE on the $\mathrm{ECN} \times$ PS interaction term $(-.034)$ and the regression of YCU on TASE (-.276). The $S E$ of the mediated moderation effect in this example is .004 , or $\left[(-.0342)\left(.041^{2}\right)+\right.$ $\left.\left(-.276^{2}\right)\left(.016^{2}\right)\right]^{1 / 2}$. The mediated moderation effect was significant $\left[a_{3} b=.009\right.$ (.004), $\left.Z=2.05, p<.05\right]$. The mediated effect-ECN (predictor) on YCU (outcome) through TASE (mediator) - differed as a function of the level of peer smoking (moderator).

Simple mediation effects (Tein et al., 2004) are then conducted to assess the significance of mediated effects across selected levels of peer smoking (i.e., different numbers of friends who smoke). First, simple slope analyses (see, e.g., Aiken \& West, 1991) showed that higher levels of identification with ECN led to increased TASE for youth with no friends who smoked $\left[a_{0}\right.$ friends $=.260$ (.035), $t=7.48]$, for youth with one friend who smoked $\left[a_{1}\right.$ friend $\left.=.227(.029), t=7.93\right]$, and for youth with three friends who smoked $\left[a_{3}\right.$ friends $\left.=.160(.039), t=4.14\right]$. These estimates and $S E$ s were then used to test for mediation $(\mathrm{ECN} \rightarrow \mathrm{TASE} \rightarrow \mathrm{YCU})$ at selected levels of peer smoking status. Significant mediation was found for the $\mathrm{ECN} \rightarrow \mathrm{TASE} \rightarrow \mathrm{YCU}$ process for youth who had no friends who smoked $[a b$ for 0 friends $=-.071$ (.014), $Z=$ -5.01 ], for youth who had one friend who smoked [ $a b$ for 1 friend $=-.062(.012), Z=-5.12]$, and for youth with three friends who smoked $[a b$ for 3 friends $=-.04$ (.01), $Z=-3.53]$. As the number of friends who smoked increased, the mediated influence of ECN on cigarette use remained significant, although it decreased.

\section{DISCUSSION}

In this study, we have extended mediation methods to include moderational effects, evaluated the multivariate delta variance estimator (Sobel, 1982) for the evaluation of the mediated moderation effect, and demonstrated mediated moderation modeling using a practical example from the adolescent tobacco literature. An example illustrated mediated moderation model estimation and tests for simple mediation effects.

The simulation results suggest that adapting this method to test mediated moderation effects is reasonable under the circumstances examined in this study, as evidenced by the lack of bias in point estimation and $S E$ estimation. Although several factors accounted for variation in RB in the point estimator and $S E$ estimator of the mediated moderation effect, RB did not reach the problematic range (i.e., $\pm 10 \%$ ) for any combination of parameters and sample sizes.

Overall, Type I error rates for this method of estimating mediated moderation effects were lower than the nominal rate of .05 , particularly for sample sizes that were below 1,000 . This is consistent with other simulation work with the use of this estimator in three-variable mediation models (MacKinnon, Lockwood, et al., 2002). Since conditions such as correlations between variables that make up the interaction term [i.e., $\mathrm{C}(X, Z)$ ] and nonzero direct effects (i.e., partial mediation) accounted for variability in Type I errors, there may be conditions that make this approach less conservative across conditions that have not been examined in previous studies.

Power generally reached $80 \%$ for larger effect size combinations (i.e., $\alpha_{3}=.36 / \beta \geq .36$ ) at $N=100$, whereas for most effects power did not reach .8 until at least $N=500$ or greater. There were also several influences on variation in power to detect mediated moderation effects, including, not surprisingly, sample size and effect size. Of particular note in this study was the identification of conditions that compromised power (when aggregated across effect sizes). Power was severely compromised when direct effects were nonzero and when the correlation between $X$ and $Z$ was nonzero. This suggests that the research contexts in which detection of mediated moderation effects may be most difficult would be nonexperimental contexts (in which component variables of interaction terms are more likely to be correlated) where partial mediated moderation (i.e., remaining direct interaction effects) occurs.

This simulation study has several limitations, including the use of nonnegative population parameter values and the use of continuous outcomes. Models such as those used to examine the interaction of risk and protective factors (e.g., positive and negative covariances) may differ in power to detect effects within mediated moderation models, because the impact of combinations of covariances that vary in magnitude and sign on parameter estimates may be less predictable. Mediation simulations have shown discrepancies between results for categorical and continuous predictors for some mediated effect estimators (MacKinnon et al., 1995), and point estimation accuracy using unstandardized mediated effect estimates with categorical outcomes can be problematic (MacKinnon \& Dwyer, 1993).

Also, use of the standard deviation of the empirical distribution of the mediated moderation effect as the true value (against which the performance of Sobel's, 1982, variance estimator is compared) presumes that the variance of the empirical sampling distribution is a sufficient statistic to describe this distribution. Recent work has suggested that the use of the mediated moderation effect variance estimator, as derived via the multivariate delta method, may be problematic in terms of the assumption of normality that has been imposed on the sampling dis- 
tribution of the mediated effect (MacKinnon, Lockwood, \& Williams, 2004). Several authors have shown that the product of two random variables is not normally distributed (Aiken \& West, 1991; MacKinnon et al., 2004; Meeker, Cornwell, \& Aroian, 1981). Simulation work has shown that the sampling distribution of the mediated effect is normal only in special cases in which the ratios of the estimate to the $S E$ for both paths that constitute the mediated effect are very large (MacKinnon et al., 2004). In this case, the confidence interval is too large, and the likelihood of rejecting a true null hypothesis that $\alpha \beta=0$ in the population is less than the nominal Type I error rate (e.g., $\approx .01$ ) in most cases for this method (MacKinnon, Lockwood, et al., 2002). In this case, this simulation project represents an evaluation of a method that appears to be conservative.

The adaptation of the mediated effect variance estimator (Sobel, 1982) for the mediation of interaction effects appears to be a useful tool that combines the strengths of both mediation and moderation frameworks. In the program evaluation context, this method may allow for the examination of differential effects of a program as a function of preexisting risk or protective factors as well as subgroup differences. This method may also help identify factors that buffer or exacerbate mediational processes in etiological research. Subsequently, researchers can identify the levels of the moderator at which a given mediational process does or does not occur (see, e.g., Morgan-Lopez et al., 2003; Tein et al., 2004). However, given some of the noted limitations, further evaluation of this approach is necessary in order to assess its utility across a wider range of research contexts.

\section{REFERENCES}

AiKen, L. S., \& WeSt, S. G. (1991). Multiple regression: Testing and interpreting interactions. Newbury Park, CA: Sage.

BARON, R. M., \& KenNy, D. A. (1986). The moderator-mediator variable distinction in social psychological research: Conceptual, strategic, and statistical considerations. Journal of Personality \& Social Psychology, 51, 1173-1182.

BENTLER, P. M. (1995). EQS structural equations program manual. Los Angeles: Multivariate Software.

Bishop, Y. M. M., Fienberg, S. E., \& Holland, P. W. (1975). Discrete multivariate analysis: Theory and practice. Cambridge, MA: MIT Press.

Bollen, K. A. (1989). Structural equations with latent variables. New York: Wiley.

Castro, F. G., Coe, K., Gutierres, S., \& Saenz, D. (1996). Designing health promotion programs for Latinos. In P. M. Kato \& T. Mann (Eds.), Handbook of diversity issues in health psychology (pp. 319345). New York: Plenum.

Castro, F. G., De Anda, M. E., Abeita, L. A., \& Morgan-Lopez, A. A. (1999, August). Healthy lifestyle I: Culturally-oriented tobacco prevention with Mexican American youth. Paper presented at the 107th meeting of the American Psychological Association, Boston.

Chen, H.-T. (1990). Theory-driven evaluations. Newbury Park, CA: Sage.

Donaldson, S. I. (2001). Mediator and moderator analysis in program development. In S. Sussman (Ed.), Handbook of program development for health behavior research and practice (pp. 470-496). Thousand Oaks, CA: Sage.

JAMES, L. R., \& BRETT, J. M. (1984). Mediators, moderators and tests for mediation. Journal of Applied Psychology, 69, 307-321.

Johnson, R. A., \& Wichern, D. W. (2002). Applied multivariate statistical analysis. Upper Saddle River, NJ: Prentice Hall.
JöresKog, K. G., \& Sörbom, D. (1996). LISREL 8 user's reference guide. Chicago: Scientific Software International.

LIPSEY, M. W. (1993). Theory as method: Small theories of treatments. In L. B. Sechrest \& A. G. Scott (Eds.), Understanding causes and generalizing about them (pp. 5-38). San Francisco: Jossey-Bass.

MacKinnon, D. P. (1994). Analysis of mediating variables in prevention intervention studies. In A. Cazares \& L. A. Beatty (Eds.), Scientific methods for prevention intervention research (DHHS Publication No. 94-3631, pp. 127-153). Washington, DC: U.S. Department of Health and Human Services.

MacKinnon, D. P., \& Dwyer, J. H. (1993). Estimating mediated effects in prevention studies. Evaluation Review, 17, 144-158.

MacKinnon, D. P., Johnson, C. A., Pentz, M. A., Dwyer, J. H., Hansen, W. B., Flay, B. R., \& Wang, E. Y. (1991). Mediating mechanisms in a school-based drug prevention program: First-year effects of the Midwestern Prevention Project. Health Psychology, 10, 164-172.

MacKinnon, D. P., Lockwood, C. M., Hoffman, J. M., West, S. G., \& SheETS, V. (2002). A comparison of methods to test mediation and other intervening variable effects. Psychological Methods, 7, 83-104.

MacKinnon, D. P., Lockwood, C. M., \& Williams, J. (2004). Confidence limits for the indirect effect: Distribution of the product and resampling methods. Multivariate Behavioral Research, 39, 99-128.

MacKinnon, D. P., Taborga, M. P., \& Morgan-Lopez, A. (2002). Mediation designs for tobacco prevention research. Drug \& Alcohol Dependence, 68(Suppl. 1), S69-S83.

MacKinnon, D. P., Warsi, G., \& Dwyer, J. H. (1995). A simulation study of mediated effect measures. Multivariate Behavioral Research, 30, 41-62.

Meeker, W. Q., JR., Cornwell, L. W., \& Aroian, L. A. (1981). The product of two normally distributed random variables (Selected Tables in Mathematical Statistics, Vol. 7). Providence, RI: American Mathematical Society.

Morgan-Lopez, A. A., Castro, F. G., Chassin, L., \& MacKinnon, D. P. (2003). A mediated moderation model of cigarette use among Mexican American youth. Addictive Behaviors, 28, 583-589.

Oetting, E. R., Donnermeyer, J. F., Trimble, J. E., \& Beauvais, F. (1998). Primary socialization theory: Culture, ethnicity, and cultural identification. The links between culture and substance use: IV. Substance Use \& Misuse, 33, 2075-2107.

SAS Institute (2000). SAS (Version 8e) [Computer software]. Cary, NC: Author

Sharma, S., Durand, R. M., \& Gur-Arie, O. (1981). Identification and analysis of moderator variables. Journal of Marketing Research, 18, 291-300.

Simon, H. A. (1954). Spurious correlation: A causal interpretation. Journal of the American Statistical Association, 49, 467-479.

Sobel, M. E. (1982). Asymptotic confidence intervals for indirect effects in structural equation models. In S. Leinhardt (Ed.), Sociological methodology (pp. 290-313). San Francisco: Jossey-Bass.

Tein, J.-Y., Sandler, I. N., MacKinnon, D. P., \& Wolchik, S. A. (2004). How did it work? Who did it work for? Mediation in the context of a moderated prevention effect for children of divorce. Journal of Consulting \& Clinical Psychology, 72, 617-624.

Tein, J.-Y., SAndLer, I. N., \& Zautra, A. J. (2000). Stressful life events, psychological distress, coping, and parenting of divorced mothers: A longitudinal study. Journal of Family Psychology, 14, 27-41.

Unger, J. B., Cruz, T. B., Rohrbach, L. A., Ribisl, K. M., BaezcondeGarbanti, L., Chen, X., ET AL. (2000). English language use as a risk factor for smoking initiation among Hispanic and Asian American adolescents: Evidence for mediation by tobacco-related beliefs and social norms. Health Psychology, 19, 403-410.

West, S. G., \& AIKEN, L. S. (1997). Toward understanding individual effects in multicomponent prevention programs: Design and analysis strategies. In K. J. Bryant, M. Windle, \& S. G. West (Eds.), The science of prevention: Methodological advances from alcohol and substance abuse research (pp. 167-209). Washington, DC: American Psychological Association.

Wolchik, S. A., West, S. G., Westover, S., Sandler, I. N., Martin, A., Lustig, J., ET AL. (1993). The children of divorce parenting intervention: Outcome evaluation of an empirically based program. American Journal of Community Psychology, 21, 293-331. 
APPENDIX A

Population Parameter Derivations

To illustrate the procedure used to derive population parameters, consider the following correlation matrix:

\begin{tabular}{lccccc} 
& $X$ & $Z$ & $X Z$ & $M$ & $Y$ \\
\hline$X$ & 1 & & & & \\
$Z$ & 0 & 1 & & & \\
$X Z$ & 0 & 0 & 1 & & \\
$M$ & .14 & .14 & .14 & 1 & \\
$Y$ & .14 & .14 & .14 & .14 & 1 \\
\hline
\end{tabular}

This matrix has uncorrelated exogenous terms, all small effects (unpartialed $r^{2}=.02$ ) between all combinations of exogenous and endogenous variables, and a small effect between the two endogenous variables. The model from Equations 3 and 4 is fit to these data. In this case, the number of unique elements in the data matrix being analyzed is the same as the number of parameters being estimated. As such, estimates of the model in Equations 3 and 4 will allow the model-implied correlation matrix and the input matrix to match perfectly. In the above case, the parameters that allow for perfect recovery of the input matrix are $\alpha_{1}=.14, \alpha_{2}=.14, \alpha_{3}=.14, \beta=.09, \tau_{1}^{\prime}=.13, \tau_{2}^{\prime}=.13$, and $\tau_{3}^{\prime}=.13$. The model in Equations 3 and 4 is simulated with the population parameters above or "true values." In this example, our interest would be in the behavior of the product estimates of $a_{3}$ and $b$, which equals .0126 , under different conditions (e.g., sample size, direct effects, and correlations between $X$ and $Z$ ). However, as was noted above, the small correlations that correspond to $\mathrm{C}(X Z, M)$ and $\mathrm{C}(M, Y)$, even if they were held constant at .14, will produce different parameter estimates for $a_{3}$ and $b$ contingent on changes in the surrounding effect sizes (e.g., direct effects). As an illustration, the model-implied covariance structure for $\mathrm{C}(X Z, M)$ is

$$
\alpha_{1} \mathrm{C}(X Z, X)+\alpha_{2} \mathrm{C}(X Z, Z)+\alpha_{3} \operatorname{Var}(X Z) .
$$

In cases of bivariate symmetry and large samples, this expression reduces to $\alpha_{3} \operatorname{Var}(X Z)$ because the expected values of $\mathrm{C}(X Z, Z)$ and $\mathrm{C}(X Z, X)$ are zero when predictors are centered (Aiken \& West, 1991).

In order to verify the accuracy of the derived covariance structure equations, population parameters were specified for all structural paths, predictor variances, predictor covariances, and error variances for the mediator $M$ and the dependent variable $Y$ (e.g., $\alpha_{1}=.14, \alpha_{2}=.14, \alpha_{3}=.14, \beta=.09, \tau_{1}^{\prime}=.13, \tau_{2}^{\prime}=.13, \tau_{3}^{\prime}=.13$ ). These parameters were then used to reproduce each element of the variance/covariance matrix using a matrix program written in SAS IML on the basis of the following matrix formula for the model-implied covariance matrix in observed path models (Bollen, 1989, p. 104).

$$
\sum(\theta)=\left[\begin{array}{cc}
(\mathbf{I}-\mathbf{B})^{-1}\left(\boldsymbol{\Gamma} \boldsymbol{\Phi} \Gamma^{\prime}+\boldsymbol{\Psi}\right)(\mathbf{I}-\mathbf{B})^{-1^{\prime}} & (\mathbf{I}-\mathbf{B})^{-1} \boldsymbol{\Gamma} \boldsymbol{\Phi} \\
\boldsymbol{\Phi} \Gamma^{\prime}(\mathbf{I}-\mathbf{B})^{-1^{\prime}} & \boldsymbol{\Phi}
\end{array}\right],
$$

where $\mathbf{I}=Y \times Y$ identity matrix; $\mathbf{B}=Y \times Y$ matrix of path coefficients among the $Y \mathrm{~s} ; \boldsymbol{\Gamma}=$ a $Y \times X$ matrix of path coefficients from the $X \mathrm{~s}$ to the $Y_{\mathrm{s}} ; \boldsymbol{\Phi}=$ an $X \times X$ matrix of covariances among the $X \mathrm{~s}$; and $\boldsymbol{\Psi}=$ a $Y \times Y$ matrix of residual covariances among the $Y$ s. 


\section{APPENDIX B \\ Normal Equations for Ordinary Least Squares Loss Functions With Respect to $\alpha_{3}$ and $\beta$}

In this appendix, the ordinary least squares (OLS) loss functions are included in order to demonstrate the influences of parameters and variances/covariances in the estimation of the elements that compose the mediated moderation effect. Partial derivatives for each model with respect to each of the relevant parameters follow.

\section{Equation 3}

$$
\begin{aligned}
& M=\alpha_{1} X+\alpha_{2} Z+\alpha_{3} X Z+\varepsilon_{1} . \\
& \mathrm{F}_{\mathrm{OLS}}=\sum\left[M-\left(\alpha_{1} X+\alpha_{2} Z+\alpha_{3} X Z\right)\right]^{2} \\
& \mathrm{~F}_{\mathrm{OLS}} \text { expanded }=\sigma^{2}(M)-2 \alpha_{1} \sigma(X, M)+\alpha_{1}^{2} \sigma^{2}(X)-2 \alpha_{3} \sigma(X Z, M)+2 \alpha_{1} \alpha_{3} \sigma(X Z, X)+\alpha_{3}^{2} \\
& \qquad \sigma^{2}(X Z)-2 \alpha_{2} \sigma(Z, M)+2 \alpha_{1} \alpha_{2} \sigma(X, Z)+2 \alpha_{2} \alpha_{3} \sigma(X Z, Z)+\alpha_{2}^{2} \sigma^{2}(Z) \\
& \partial \mathrm{F}_{\mathrm{OLS}} / \partial \alpha_{3}=2 \sigma(X Z, M)+2 \alpha_{1} \sigma(X Z, X)+2 \alpha_{3} \sigma^{2}(X Z)+2 \alpha_{2} \sigma(X Z, Z) \\
& \partial \mathrm{F}_{\mathrm{OLS}} / \partial \alpha_{3} \operatorname{minimum}=\frac{\sigma(X Z, M)-\alpha_{1} \sigma(X Z, X)-\alpha_{2} \sigma(X Z, Z)}{\sigma^{2}(X Z)} .
\end{aligned}
$$

\section{Equation 4}

$$
\begin{aligned}
& Y=\beta M+\tau_{1}^{\prime} X+\tau_{2}^{\prime} Z+\tau_{3}^{\prime} X Z+\varepsilon_{2} . \\
& \mathrm{F}_{\mathrm{OLS}}=\Sigma\left[Y-\left(\tau_{1}^{\prime} X+\tau_{2}^{\prime} Z+\tau_{3}^{\prime} X Z\right)\right]^{2} \\
& \mathrm{~F}_{\mathrm{OLS}} \text { expanded }=\sigma^{2}(Y)+\beta^{2} \sigma^{2}(M)+2 \beta \tau_{1} \sigma(X, M)+\tau_{1}^{2} \sigma^{2}(X)+2 \beta \tau_{3} \sigma(X Z, M)+2 \tau_{1} \tau_{3} \sigma(X Z, X)+\tau_{3}^{2} \sigma^{2}(X Z) \\
& -2 \beta \sigma(M, Y)-2 \tau_{1} \sigma(X, Y)-2 \tau_{3} \sigma(X Z, Y)+2 \beta \tau_{2} \sigma(Z, M)+2 \tau_{1} \tau_{2} \sigma(X, Y)+2 \tau_{2} \tau_{3} \sigma(X Z, Z) \\
& -2 \tau_{2} \sigma(Z, Y) Z+\tau_{2}^{2} \sigma^{2}(Z) \\
& \partial \mathrm{F}_{\mathrm{OLS}} / \partial \beta=2 \beta \sigma^{2}(M)+2 \tau_{1} \sigma(X, M)+2 \tau_{3} \sigma(X Z, M)-2 \sigma(M, Y)+2 \tau_{2} \sigma(Z, M) \\
& \partial \mathrm{F}_{\mathrm{OLS}} / \partial \beta \text { minimum }=\frac{\tau_{1} \sigma(X, M)-\tau_{3} \sigma(X Z, M)+\sigma(M, Y)-\tau_{2} \sigma(Z, M)}{\sigma^{2}(M)}
\end{aligned}
$$

